\title{
Bevacizumab Improves Achilles Tendon Repair in a Rat Model
}

\author{
Herbert Tempfera,b Alexandra Kaser-Eichberger ${ }^{c} \quad$ Christine Lehnera,b \\ Renate Gehwolf ${ }^{a, b}$ Stefanie Korntner ${ }^{a, b}$ Nadja Kunkel ${ }^{a, b} \quad$ Andrea Wagner ${ }^{a, b}$ \\ Moritz Gruetz ${ }^{a, b} \quad$ Ludwig M. Heindle Falk Schroedll, ${ }^{c / d}$ Andreas Traweger ${ }^{a, b}$ \\ aInstitute for Tendon and Bone Regeneration, Spinal Cord Injury and Tissue Regeneration Center \\ Salzburg, Paracelsus Medical University, Salzburg, ${ }^{b}$ Austrian Cluster for Tissue Regeneration, Vienna, \\ 'Dept. Ophthalmology/ Optometry, Research Program Experimental Ophthalmology, Paracelsus \\ Medical University Salzburg, 'Department of Anatomy, Paracelsus Medical University Salzburg, Austria, \\ eDepartment of Ophthalmology, University of Cologne, Cologne, Germany
}

\section{Key Words}

Tendon regeneration - Antiangiogenic treatment - Biomechanics - Rat tendon defect model - VEGF-A

\begin{abstract}
Background/Aims: Effective wound-healing generally requires efficient re-vascularization after injury, ensuring sufficient supply with oxygen, nutrients, and various cell populations. While this applies to most tissues, tendons are mostly avascular in nature and harbor relatively few cells, probably contributing to their poor regenerative capacity. Considering the minimal vascularization of healthy tendons, we hypothesize that controlling angiogenesis in early tendon healing is beneficial for repair tissue quality and function. Methods: To address this hypothesis, Bevacizumab, a monoclonal antibody blocking VEGF-A signaling, was locally injected into the defect area of a complete tenotomy in rat Achilles tendon. At 28 days postsurgery, the defect region was investigated using immunohistochemistry against vascular and lymphatic epitopes. Polarization microscopy and biomechanical testing was used to determine tendon integrity and gait analysis for functional testing in treated vs non-treated animals. Results: Angiogenesis was found to be significantly reduced in the Bevacizumab treated repair tissue, accompanied by significantly reduced cross sectional area, improved matrix organization, increased stiffness and Young's modulus, maximum load and stress. Further, we observed an improved gait pattern when compared to the vehicle injected control group. Conclusion: Based on the results of this study we propose that reducing angiogenesis after tendon injury can improve tendon repair, potentially representing a novel treatment-option.
\end{abstract}




\section{Cellular Physiology Cell Physiol Biochem 2018;46:1148-1158 \\ \begin{tabular}{l|l} 
and Biochemistry Publis. 10.1159/000489057 & $\begin{array}{l}\text { (c) } 2018 \text { The Author(s). Published by S. Karger AG, Basel } \\
\text { www.karger.com/cpb }\end{array}$
\end{tabular} \\ Tempfer et al.: Bevacizumab Improves Tendon Repair}

\section{Introduction}

Tendon pathologies affect both the working population and active as well as recreational athletes, placing an enormous socioeconomic burden on healthcare systems worldwide. Therapeutic interventions remain limited and acute tendon injuries and chronic tendinopathies remain clinically challenging, mostly being addressed surgically or by a conservative approach. Generally, tendon healing is a slow, ineffective process often resulting in the formation of a scar tissue with inferior quality, compromising the mechanical function [1].

Healthy adult tendons are characterized by a low cell density and are sparsely vascularized [2]. Achilles tendons are supplied with vessels originating from the osteotendineous junction, the musculotendinous junction and the mesotenon/ peritenon surrounding the tendon. From the latter structure, vessels penetrate the tendon, entering the endotenon sheaths [3]. However, in the human Achilles tendon a zone of relative avascularity was described $2-6 \mathrm{~cm}$ proximal from the bone insertion [4]. In contrast, in degenerated tendons or after injury an increase in vascularization is observed, which is considered a clinical sign of tendinopathy. Therefore, although neovascularization is an indispensable process during the regeneration of virtually any tissue, it potentially has a negative impact on functional tendon repair [5].

Interestingly, in healthy tendons blood vessels are relatively tight, establishing a sizeselective interface and restricting the free passage of blood-borne components into the tendon proper [6]. This protection is essential, as we have previously shown that the presence of serum is required for tendon stem/progenitor cell differentiation, particularly towards the osteogenic lineage. Moreover, serum contact causes upregulation of the matrix degrading enzymes MMP2 and MMP9. Thus, excessive supply with blood components may contribute to unfavorable processes following tendon injury, such as erroneous cell differentiation, disturbance of matrix organization and concomitant impairment of mechanical function [6].

In normal tendon healing/ repair tissue formation after injury, an inflammatory phase is followed by a phase of cell proliferation and matrix deposition, finally tissue remodeling may occur, leading to a more or less functional repair tissue. Particularly in the proliferative phase, neoformation of blood vessels occurs, leading to a hypervascular repair tissue [7]. In a canine model of tendon repair, VEGF expression was found to peak at 7 days post injury, an early time point in the proliferative phase of tendon repair [8]. Neovascularization is also commonly observed in tendinopathic tendon, as a response to overuse microtrauma and/or local inflammation. This vessel in-growth is likely to be associated with erroneous innervation, which in turn causes pain [7].

To restore tendon function, most treatments are believed to favor neovascularization, such as the application of autologous platelet rich plasma or ultrasound guided needle fenestration [9] or the application of pulsed extracorporeal shockwaves [4]. However, it is known from other well vascularized tissues, that neovascularization can also be detrimental, leading to undesired effects. For example in the eye, retinal neovascularization is the main pathogenic process underlying age-related macular degeneration, diabetic retinopathy, or diabetic macular edema resulting in progressive visual loss. Here, newly formed vessels are compromised and show increased vascular permeability leading to diffuse leakage from capillary walls [10] resulting in intraretinal hemorrhage and edema. In skin wounds, VEGF-A mediated neovascularization is a prerequisite for tissue healing, however it has been shown that excessive production of VEGF also can result in excessive scar formation. In this context it has been shown that reducing VEGF signaling leads to improved skin regeneration. Moreover, scarless healing of fetal skin wounds is strongly associated with lower levels of VEGF [11]. As a large body of evidence supports a key pathogenic role of VEGF-A in neovascularization we hypothesize that reduction of neo-vessel formation by injection of Bevacizumab (Avastin), a recombinant humanized monoclonal antibody that inhibits VEGF-A signaling, can improve tendon repair. To this end, we performed a complete tenotomy in the rat Achilles tendon and show that application of Bevacizumab not only reduces neoangiogenesis, but also improves the structure and function of the newly formed tendon repair tissue in early 


\section{Cellular Physiology Cell Physiol Biochem 2018;46:1148-1158 and Biochemistry Published online: April 19, $2018 \quad \begin{aligned} & \text { DOI: 10.1159/000489057 } 2018 \text { The Author(s). Published by S. Karger AG, Basel } \\ & \text { www.karger.com/cpb }\end{aligned}$}

Tempfer et al.: Bevacizumab Improves Tendon Repair

tendon healing by immunohistochemistry, polarization microscopy, biomechanical testing and gait analysis at 14 and 28d post-surgery.

\section{Materials and Methods}

\section{Animals and surgical procedure}

All animal experiments and procedures were conducted in accordance with Austrian laws and were approved by Austrian regulatory authorities on animal experimentation (permit number BMWFW66.019/0012-WF/V/3b/2015). A total of 58 female Lewis rats aged 2 months were included in the study (Table 1). A $2 \mathrm{~mm}$ full-thickness transverse Achilles tenotomy was created on the right hind limb of each animal. The tendon was transversally excised and 2 $\mathrm{mm}$ in proximal/distal extension were removed $3 \mathrm{~mm}$ proximal of the calcaneus resulting in a complete separation of the proximal and distal segments. Tendons were left in place without further adaption and only the skin was sutured [12].

For better retention, Bevacizumab $(25 \mathrm{mg} / \mathrm{ml}$, Avastin, Roche, Germany) was mixed with BD Puramatrix ${ }^{\mathrm{TM}}$ Peptide Hydrogel (BD Biosciences, Bedford, USA) at a ratio of 3:2 (v/v). At 4 and 11 days post-surgery, $3 \mathrm{x} 25 \mu \mathrm{l}$ of Bevacizumab/Puramatrix ${ }^{\mathrm{TM}}$ were injected into the defect site at 5, 10 and $15 \mathrm{~mm}$ proximal to the calcaneus, the control group received similar injections with Puramatrix ${ }^{\mathrm{TM}} /$ saline. For the $^{2}$ injection, animals were briefly anesthetized by isoflurane inhalation.

\section{Tissue harvest and size measurements}

2 and 4 weeks after surgery, animals were euthanized by intracardial injection of barbiturate and both Achilles tendons were dissected. Before preparation of the tendons, tendon thickness was measured using a digital caliper $28 \mathrm{~d}$ post-surgery ( $\mathrm{n}=26$ intact tendons, $\mathrm{n}=13$ vehicle group, $\mathrm{n}=13$ Bevacizumab group). Thickness was determined $3 \mathrm{~mm}$ proximal of the calcaneus, both in medial-lateral and anterior- posterior direction. Cross-sectional area (CSA) was calculated assuming an elliptic shape. For all analyses involving intact tendons, the contralateral legs were used.

\section{Histologic analyses}

Preparation of tissue sections. For histologic analyses, Achilles tendon repair tissues and contralateral tendons (n=3/group 2 weeks, n=5/group 4 weeks) were dissected from the proximal musculotendinous junction to the distal tendon-to-bone attachment zone and processed as described earlier [13]. Samples were fixed in $4 \%$ paraformaldehyde for $12 \mathrm{~h}$ at $4^{\circ} \mathrm{C}$ and after $12 \mathrm{~h}$ washing in phosphate buffered saline at $4^{\circ} \mathrm{C}$ embedded in cryomedium (Surgipath Cryogel ${ }^{\circledR}$, Leica Microsystems, Vienna, Austria). Subsequently, $12 \mu \mathrm{m}$ cryosections were prepared (Leica CM1950, Leica, Austria).

Immunohistochemistry. After a 5 min rinse in tris-buffered saline (TBS; Roth, Karlsruhe, Germany) slides were incubated for $1 \mathrm{~h}$ at RT in TBS containing 10\% donkey serum (Sigma-Aldrich, Wien, Austria), $1 \%$ bovine serum albumin (BSA; Sigma-Aldrich, Vienna, Austria), and 0.5\% Triton X-100 (Merck, Darmstadt, Germany). Followed by a 5 min rinse, slides were subsequently incubated for double or triple immunohistochemistry (overnight at $4^{\circ} \mathrm{C}$ ) with antibodies against rat endothelial cell antigen (RECA-1 \#AB9774, Abcam, Cambridge, UK; 1:500), CD68 (\#sc20060, Santa Cruz, Dallas, USA; 1:50), lymphatic vessel endothelial hyaluronan receptor 1 (LYVE-1; Acris; Herford, Germany; 1: 50) and podoplanin (R\&D Systems/ Biotech, Abingdon, UK; 1: 50) all diluted in TBS, BSA, and Triton X-100. After a rinse in TBS (four times $5 \mathrm{~min}$ ) binding sites of primary antibodies were visualized by corresponding Alexa488-, Alexa555-, or Alexa647tagged antisera (1:1000; Invitrogen, Karlsruhe, Germany) in TBS, containing 1\% BSA and 0.5\% Triton X-100 (1 $\mathrm{h}$ at RT) followed by another rinse in TBS (four times $5 \mathrm{~min}$ ). Some of the slides received an additional nuclear staining using 4',6-Diamidino-2-phenylindol dihydrochlorid (DAPI). For that, slides were incubated $10 \mathrm{~min}$ (1:4000, stock $1 \mathrm{mg} / \mathrm{ml}$, VWR, Vienna, Austria) followed by a rinse in PBS (three times $5 \mathrm{~min}$ ). All slides were embedded in Fluoromount ${ }^{\mathrm{TM}}$ Aqueous Mounting Medium (Sigma Aldrich, Vienna, Austria) TBSglycerol (1:1 at pH 8.6). Negative controls were performed by omission of the primary antibodies during incubation and resulted in absence of immunoreactivity. 


\section{Cellular Physiology Cell Physiol Biochem 2018;46:1148-1158

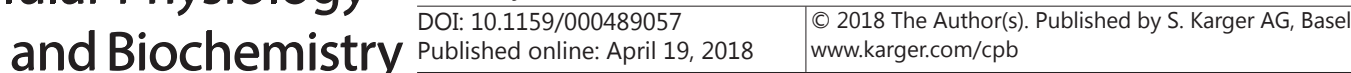 \\ Tempfer et al.: Bevacizumab Improves Tendon Repair}

Tile scan images were taken of the entire newly formed tissue ( 3 sections/ animal, 5 animals/group) and the immunopositive area was quantified using Image J software. The 3 sections of each specimen were taken with an approximate distance of $250 \mu \mathrm{m}$. Similarly, nuclear density counting was performed by counting DAPI- positive nuclei by Image J software (Cell counter plugin V.2).

To evaluate the presence of Bevacizumab after injection, it was detected by incubating the sections with a donkey anti-human IgG labeled with Texas Red (\#609-709-123, Rockland Inc, Limerick, USA 1:500) for $1 \mathrm{~h}$ at room temperature.

Histology and polarization microscopy. Histochemistry was perfomed on cryosections as described above. After staining the sections with Weigert hematoxylin for two minutes, the staining was stopped with $1 \%$ acetic acid including a short differentiation step by shortly dipping the slides into $\mathrm{HCl} / \mathrm{ethanol}$. After blueing the sections under running tap water for 10 minutes, sections were stained with $1 \%$ eosin Y solution for 1 minute and again immersed in $1 \%$ acetic acid to stop the staining reaction. Subsequently, the sections were dehydrated in an increasing ethanol series (70\%, 96\%, 2x 100\%) and incubated twice in Rotihistol. Finally, sections were coverslipped with mounting medium.

For safranin 0 staining, after a short incubation $(20 \mathrm{sec})$ in fast green solution and a quick rinse in $1 \%$ acetic acid solution for $10 \mathrm{sec}$, sections were stained in $0.1 \%$ safranin 0 solution for 5 minutes. The slides were then dehydrated through ethanol and Roti-Histol (Carl Roth, Karlsruhe, Germany) and mounted in Roti-Histokitt (Carl Roth, Karlsruhe, Germany).

For polarization microscopy, unstained sections of intact tendons and of repair tissues from the control group and the Bevacizumab-treated group ( 5 animals per group- 5 sections/ animal) were imaged using a $5 \mathrm{x}$ objective equipped with a polarization filter mounted on an Axioplan microscope (Carl Zeiss, Jena, Germany). Using this filter, organized collagen type I fibers are visualized in white color when exposed to polarized light at an angle of $45^{\circ}$. Since the strength of polarization is a surrogate of collagen organization this could be measured by analysis of the average pixel intensity (Image J software). The degree of fiber orientation was determined by measuring the average fiber angle dispersion (Image J Software, Directionality V 2.0.2 tool).

\section{Biomechanical testing}

4 weeks post-surgery/transection, Achilles tendon repair tissues as well as contralateral control tendons of the vehicle group $(n=11)$ and the Bevacizumab group $(n=11)$ were harvested and mechanical properties of these tissues were tested on a universal material testing machine (Zwick, Ulm, Germany) at a $15^{\circ}$ loading angle. The uninjured contralateral tendons were used as intact controls. Prior to loading to failure, tendons were pre-conditioned by loading to $10 \mathrm{Newton}(\mathrm{N})$ for 20 seconds and to $5 \mathrm{~N}$ for another 20 seconds ( 5 repeats) at a speed of $0.1 \mathrm{~N} / \mathrm{s}$. Maximum tensile load was defined as the maximum force at tendon failure (also expressed in $\mathrm{N}$ ). Tendon stiffness was calculated from the linear proportion of the force/ elongation curve and is expressed as $\mathrm{N} / \mathrm{mm}$. Only those tendons which ruptured intra-tendinously were included for analysis ( $n=11$ intact, $n=9$ vehicle, $n=11$ Bevacizumab), while samples failing at the clamping site were excluded. Significance between vehicle and Bevacizumab group was determined by Mann-Whitney test and considered significant if $\mathrm{p}<0.05$.

\section{Gait analysis:}

The rats were placed in a custom made treadmill, forcing the animals to run at a speed of $0.3 \mathrm{~m} / \mathrm{s}$. The gait was recorded using a GoPro Hero4 Session camera with a frame rate of 100 frames per second. Single frames were extracted and the ankle joint angles at mid-stance and pre-swing were analyzed. For that, 5 steps were measured ( $n=7$ animals / group) using Image J software. For statistical analysis, Students t-tests were performed to compare groups.

\section{Results}

All animals showed normal behavior until the planned end points at $14 \mathrm{~d}$ and $28 \mathrm{~d}$ postsurgery, with non-restricted weight bearing 24 hours after surgery. 


\section{Cellular Physiology Cell Physiol Biochem 2018;46:1148-1158

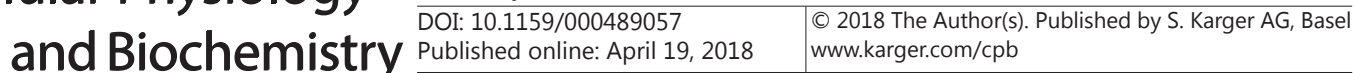 Tempfer et al.: Bevacizumab Improves Tendon Repair}

Bevacizumab affects tendon crosssectional area

4 weeks after surgery, the repair tissues in the vehicle treated group showed a significantly $\quad(\mathrm{p}<0.001)$ increased cross-sectional area (CSA) $\left(9.1 \mathrm{~mm}^{2} \pm 2.0\right)$ when compared to intact, contralateral Achilles tendons $\left(1.5 \mathrm{~mm}^{2} \pm\right.$ $0.3)$. In the Bevacizumab-treated tendons, CSA was significantly $(\mathrm{p}<0.001)$ reduced $\left(5.6 \mathrm{~mm}^{2} \pm 1.8\right)$ compared to the vehicletreated tendons (Fig. $1 \mathrm{a}, \mathrm{b}$ ).

Bevacizumab reduces hem- but not lymphangiogenesis

Labelling of injected Bevacizumab with a human IgG-antibody revealed that after a single injection at $4 d$ p.o., Bevacizumab was detectable $7 \mathrm{~d}$ p.o., however not $14 \mathrm{~d}$. Upon a second injection at $11 \mathrm{~d}$ p.o., Bevacizumab was also detectable at $14 \mathrm{~d}$ p.o. (Fig. 2b-e). Using immunohistochemistry, VEGF was not detectable in intact tendon tissue, whereas VEGF presence was clearly detectable in both groups at 3, 7, 14 and $28 \mathrm{~d}$ post-surgery, with a peak at 7 days. The same pattern was observed for CD68 (Fig. 2a). Quantification of RECA-1 positive areas $28 \mathrm{~d}$ post-surgery revealed that Bevacizumab treatment significantly reduced RECA- 1 positive areas by $43.7 \%$ compared to the vehicle treated group $(1.37 \% \pm 0.30$ vs. $3.1 \% \pm 1.3$ vs ; $p<0.05)$. After 14 days, Bevacizumab treatment reduced the RECA-1-positive area by trend from $2.36 \% \pm 0.8$ in the vehicle treated animals to $0.77 \% \pm 0.4$ in the group that had received Bevacizumab ( $\mathrm{p}=0.07)$ (Fig 3a-c). In addition, the average vessel diameter was reduced by Bevacizumab from $23.15 \mu \mathrm{m} \pm 3.93$ in the vehicle group to $16.56 \mu \mathrm{m} \pm 2.23$ in the Bevacizumab group ( $\mathrm{p}=0.06)$ at $28 \mathrm{~d}$ (Fig.3e).

When applying immunohistochemistry against podoplanin for the visualization of lymphatic vessels, a significant difference in areas positive for podoplanin was not evident between vehicle- and Bevacizumab-treated animals at 28d, with $0.64 \% \pm 0.33$ podoplanin ${ }^{+}$area in the vehicle group and $0.78 \% \pm 0.22$ in the Bevacizumab group; $p=0.5$ (Fig. 3f-h).

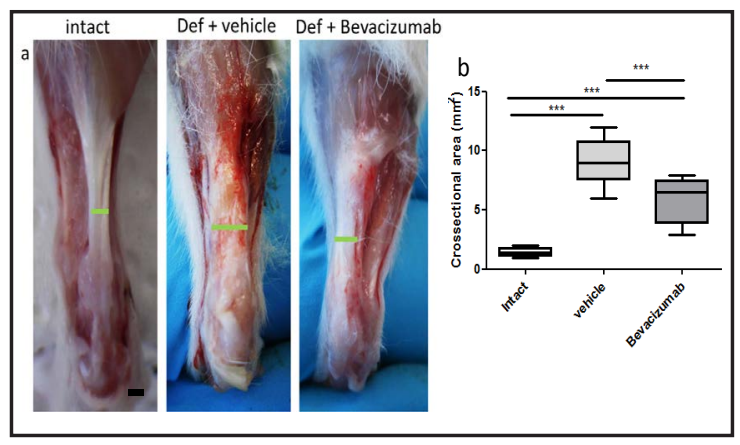

Fig. 1. Gross morphology of the Achilles tendons shows that the vehicle treated defects are thicker and more irregular than intact tendons and defects treated with Bevacizumab 28d post surgery (a) scale: $2 \mathrm{~mm}$. The cross-sectional areas (CSA) of both defect groups are significantly increased compared to intact tendon, the Bevacizumab treated defects show a significantly decreased CSA compared to the vehicle treated defects *** $\mathrm{p}<0.001, \mathrm{n}=(\mathrm{n}=26$ intact tendons, $\mathrm{n}=13$ vehicle group, $n=13$ Bevacizumab group) (b).

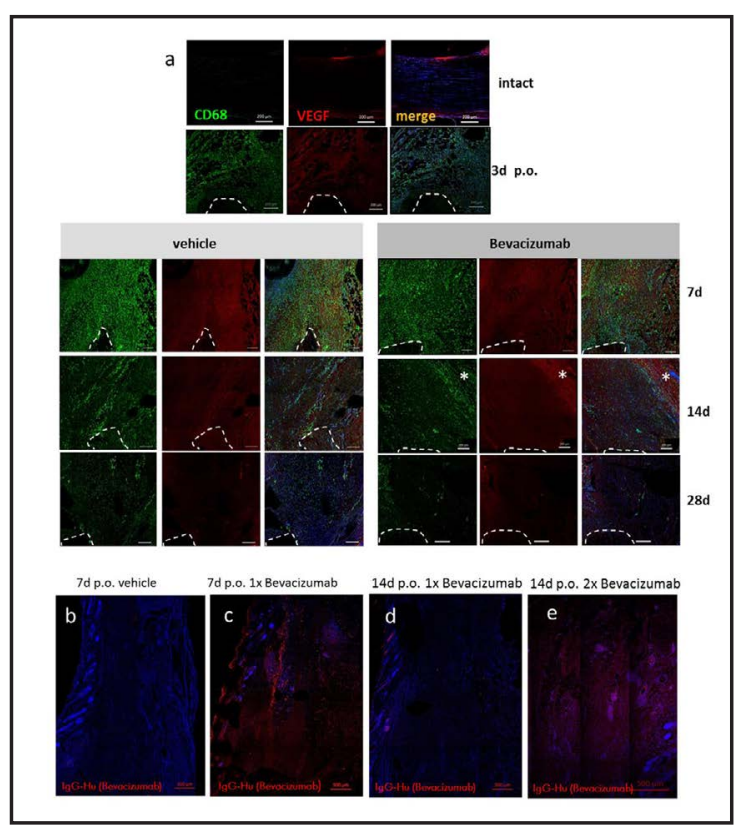

Fig. 2. Immunostainigs with CD68 and VEGF antibodies reveal an increase in expression of both markers at 3 and $7 d$ post opertion followed by a decline at $d 14$ and $\mathrm{d} 28$. Bevacizumab tratment leads to a reduction of CD68+ cells at d14 and d28 (a). (dashed line: distal tendon stump; asterisk: dermis; scale bar: $200 \mu \mathrm{m})$. Bevacizumab is detectable $3 \mathrm{~d}$ after injection at $4 \mathrm{~d}$ p.o. (c), but not at $14 \mathrm{~d}$ p.o. (d). Upon injection at 4 and $11 \mathrm{~d}$ p.o., Bevacizumab is detectable at $14 \mathrm{~d}$ p.o.(e). In the vehicle injected tendon, no Bevacizumab is detectable (b). 
Fig. 3. Quantitative analysis of immunofluorescence images shows the capability of Bevacizumab to reduce Reca1+ vessels and CD68positive cells at 14 and $28 \mathrm{~d}$ p.o. $(\mathrm{a}, \mathrm{d})$, illustrated by representative images $(b, c)$ dashed line: proximal tendon stump. By trend, also the average vessel diameter is reduced in the Bevacizumab group after 28d (e). The density of Podoplanin+ lymphatic vessels is not affected by Bevacizumab (f), as illustrated by representative images taken from the newly formed repair tissue (g,h).

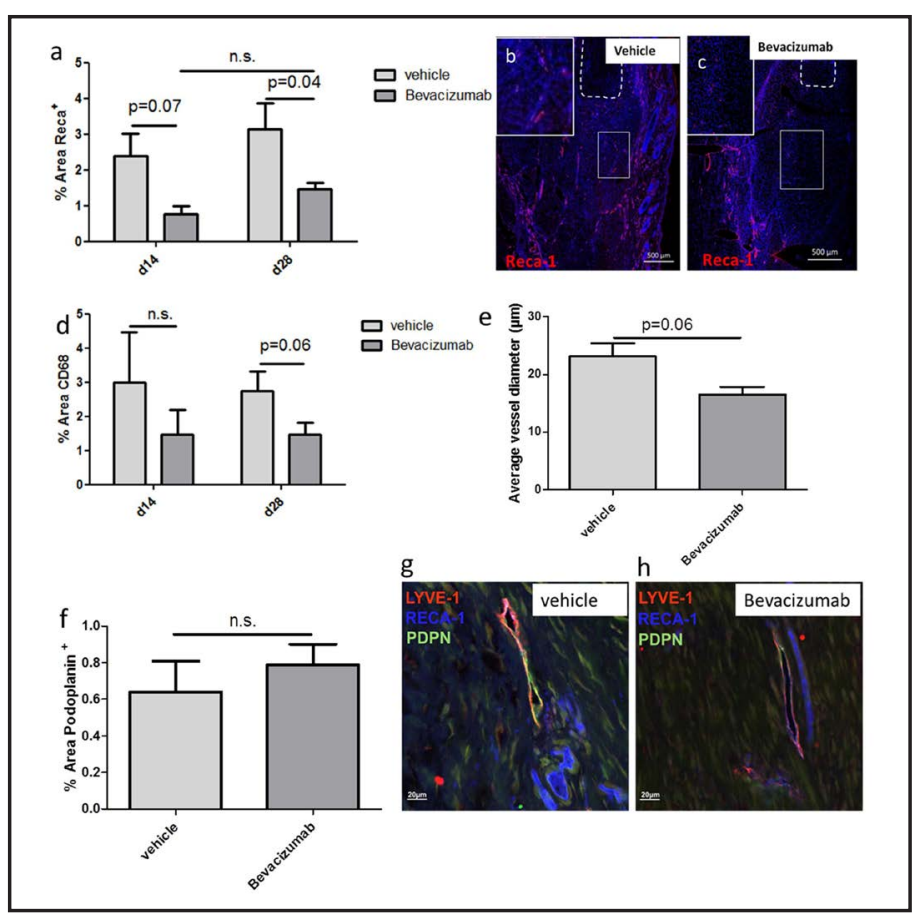

Fig. 4. Nucler density is decreased by trend in the Bevacizumab-treated animals (a). HE- staining revealed no obvious difference between the groups, with areas of aligned matrix and cells in both groups at $14 \mathrm{~d}$ p.o. (b, c) and with formation of cartilage like tissue at $28 \mathrm{~d}$ p.o. (d,e,) scale bars: $3 \mathrm{~mm}$. Safranin 0-stain confirmes the presence of chondrified areas in both groups at $28 \mathrm{~d}$ p.o. (f, scale bars $200 \mu \mathrm{m})$. Polarized light microscopy analysis of tendon sections reveal that Bevacizumab significantly decreases average fiber angle dispersion, thus leading to increased fiber orientation $(\mathrm{g})$, whereas the degree of birefringence (a measure for maturation, $y$-axis: grey scale) is unaffected (h). Shown are representative images (i, scale bar: $1 \mathrm{~mm}$ ).

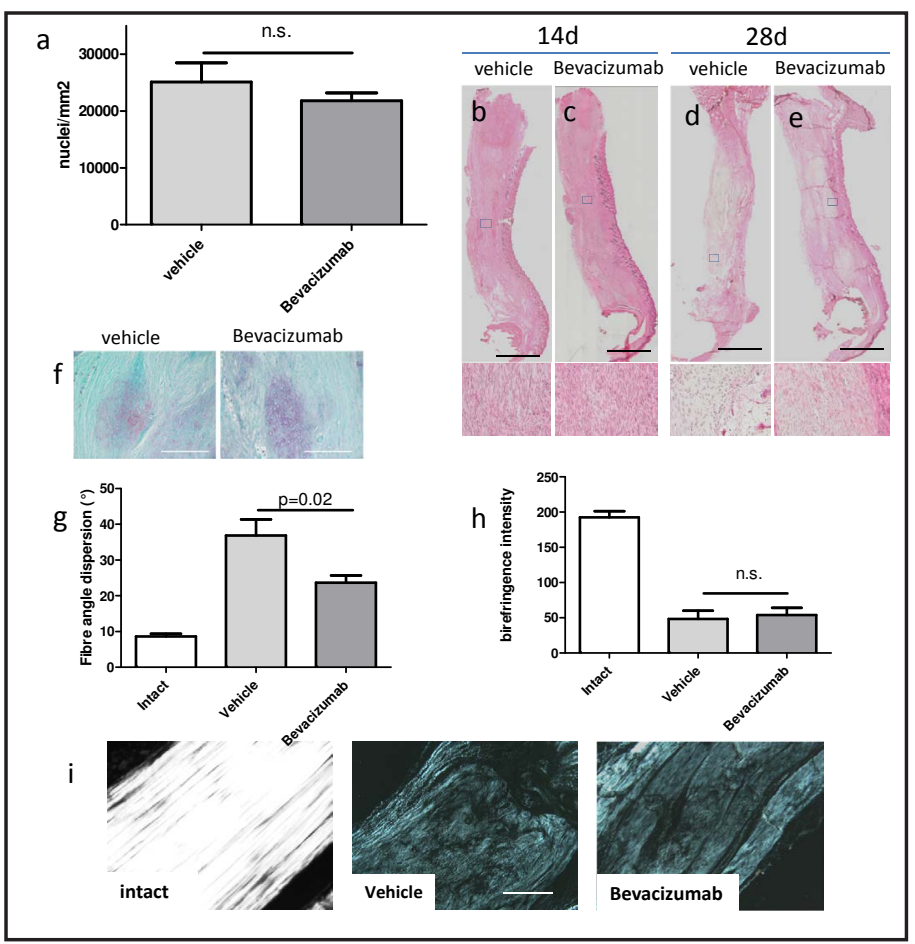

Bevacizumab improves ECM orientation

Analysis of DAPI stained sections 28d p.o. showed a reduction of nuclear density by trend in the treatment group $\left(25110 \pm 6764\right.$ cells $/ \mathrm{mm}^{2}$ in the vehicle group and $21789 \pm$ 2770 in the Bevacizumab group). (Fig. 4a)

A heterogenous tissue patterning was observed by qualitative analysis of HE- sections in both groups at $14 \mathrm{~d}$ and at $28 \mathrm{~d}$. Both groups showed partially well aligned, tendon like tissue with tendon typical crimp (Fig. 4b-e). At 28d, in both groups chondrified areas were 
detectable, as confirmed by safranin- o staining (Fig. 4f). Polarization microscopy revealed that the tendon repair tissues of Bevacizumab-treated animals displayed a higher degree of fiber orientation; $23.66^{\circ}$ average dispersion \pm 4.58 compared to $36.88^{\circ}$ average dispersion \pm 10.08 in the vehicle group ( $\mathrm{p}=0.02)$, thus being more similar to the intact tendon (Fig. 4g).

No significant difference was observed in birefringence intensity, a hallmark for collagen compactness and maturation (Fig. 4h)

Bevacizumab increases repair tissue stiffness and maximum tensile load

At 28d post-surgery, maximum tensile loads were significantly increased in the Bevacizumab group ( $47.7 \mathrm{~N} \pm 6.41$ ) compared to the vehicle group $(32.41 \mathrm{~N}$ \pm 9.23, p=0.002) (Fig. 5a). Similarly, maximum tensile stress was higher, with $9.09 \mathrm{MPa} \pm 3.20$ in the Bevacizumab animals and $4.55 \mathrm{MPa} \pm 1.97$ for the vehicle group ( $p=0.003$; Fig. 5b). Stiffness was significantly $(p=0.03)$ increased in the Bevacizumab animals $(32.14 \mathrm{~N} / \mathrm{mm} \pm$ 10.01) compared to the vehicle injected animals $(23.42 \mathrm{~N} / \mathrm{mm} \pm 5.53)$ (Fig. $5 \mathrm{c}$ ). Also, material-level properties of the repair tissue were improved by treatment with Bevacizumab, as the Young's modulus was $73.28 \mathrm{MPa} \pm 32.09$ for the treated animals and $34.81 \mathrm{MPa} \pm 10.78$ in the vehicle group ( $p=0.003$; Fig. $5 d$ ).

\section{Bevacizumab positively affects gait pattern}

Gait analysis revealed a significant $(p=0.02)$ improvement of ankle joint angle at the pre- swing phase of the gait from $60.2^{\circ} \pm 10.8$ in the vehicle treated group compared to $73.1^{\circ} \pm 9.4$ in the Bevacizumab treated group. In healthy (non-operated) animals, this angle was measured to be $83.0^{\circ} \pm 4.2$ (Fig. 6 a).

In contrast, the ankle joint angle at mid-stance was unaffected by local Bevacizumab injection ( $44.23^{\circ} \pm 10.3$ in the vehicle group and $47.9^{\circ} \pm 5.2$ in Bevacizumab group). In uninjured animals the mid stance joint angle was $32.1^{\circ} \pm 1.4$ ) (Fig. 6b).

\section{Discussion}

Chronic tendon degeneration due to long term overuse or following traumatic tendon injuries is a major clinical challenge. Besides loss of extracellular matrix organization, formation of cartilage-like tissue, fatty deposits or mineralized scar tissue and vascular 
ingrowth are hallmarks of such degenerative events [13]. Current strategies to reduce tendon degeneration and pain aim to support tissue regeneration by delivery of growth factors, for example by injection of platelet rich plasma [14], or by enforcing vascular ingrowth to improve nutrient supply. An example for the latter is the application of extracorporeal shockwaves, which are known to induce VEGF- signaling and pro-inflammatory cytokines [15]. Along these lines, adenovirus mediated VEGF gene transfer was shown to be partly beneficial in a chick model of tendon repair [16]. Also the application of recombinant VEGF has shown beneficial effects on the biomechanical properties of healing in rat models [17, 18]. However, in none of these studies enhanced vascularization due to elevation of VEGF levels has actually been demonstrated.

With our work we show that Bevacizumab is capable of reducing hemangiogenesis in tendon repair tissue and that reducing angiogenesis during early tendon repair following injury leads to improved biomechanical properties, as demonstrated by an increase in tissue stiffness and maximum tensile load. This improvement is not due to increased tendon crosssectional area, as the dimension-independent parameters maximum tensile stress and Young's modulus are also significantly increased in the Bevacizumab treated repair tissues. The fact that the Bevacizumab treated tendons show a smaller cross sectional area (Fig. 1) compared to vehicle treated ones can be interpreted as a reduction of edema and consequent scar formation. This finding is also supported by the observation that Bevacizumab treatment leads to an increase in extracellular matrix organization, a hallmark of improved tendon healing [19]. Further, to some extent, both groups showed signs of chondrification, with no detectable difference between the groups (Fig. 4b-f). The positive effect of applying Bevacizumab after an Achilles tenotomy is further substantiated by an improvement in the gait pattern of the treated animals, with treated animals more closely resembling a gait comparable to that of healthy animals. Most likely this improved gait is concomitant with the improved mechanical properties of the repair tissue, which is a prerequisite for a better range of motion.

Interestingly, a previous study suggests that anti-VEGF-A treatment may increase seemingly positive effects of platelet rich plasma for treating collagenase induced tendinopathy, mainly based on qualitative histological analysis. However, the authors did not investigate the ramifications on tendon repair on a functional level by Bevacizumabtreatment alone [20].

While the potential underlying mechanisms leading to the observed improvements are not fully understood, we demonstrate that VEGF not only regulates neoangiogenesis in wound healing, as seen by decreased vascular bed, but also directly or indirectly affects collagen fiber orientation, as demonstrated by polarization microscopy. Interestingly, in earlier studies, scarlessly healed fetal wounds in skin had significantly lower levels of VEGF than fibrotic adult wounds and similar to our findings, neutralization of VEGF leads to this reduced scar formation and improved wound healing [11]. Several mechanisms are currently being discussed to cause these effects, such as trophic effects of VEGF on myofibroblasts, increased apoptotic load due to vessel regression and alterations in vessel permeability leading to edema and delivery of growth factors [21]. However, results remain in part controversial.

VEGF-treatment is known to lead to the formation of immature capillaries [22], which is supported by our observation of reduced capillary diameter (Fig. 2d). It may also result in increased vessel permeability; we therefore investigated the effect of Bevacizumab on vascular permeability. However, we did not observe any significant differences between the treatment and control group following injection of a $10 \mathrm{kD}$ dextran tracer at $28 \mathrm{~d}$ p.o. (data not shown).

Bevacizumab is a humanized antibody with high capacity to block the interaction of VEGF with its receptor, thus inhibiting endothelial cell proliferation [23]. More precisely, Bevacizumab inhibits the interaction of VEGF-A, the isoform binding to VEGF receptors 1 and 2 . These receptors promote hemangiogenesis, whereas lymphangiogensis is controlled by VEGF receptor 3, which is not affected by Bevacizumab. Thus our observation of 
lymphangiogenesis remaining unchanged in the tendons of Bevacizumab treated animals is well in line with the known mechanisms of action of Bevacizumab [24, 25]. The efficacy of Bevacizumab to inhibit angiogenesis and tumor growth in human patients has been demonstrated in numerous tumor models, including breast, prostate, and colorectal cancer as well as rhabdomyosarcoma and Wilms' tumor [26]. As an off label use, Bevacizumab is applied intravitreally to treat age related macular degeneration with good clinical outcome and good safety $[27,28]$. The most common adverse events associated with systemically applied Bevacizumab in humans are hypertension, proteinuria, thromboembolism, bleeding, gastrointestinal perforation and prolonged wound healing [29]. Importantly, while potential systemic side effects were not explicitly addressed in our study, signs of intolerance or changed behavioral signs were not observed in any of the Bevacizumab treated animals within the period tested.

Our study has some limitations that need to be considered. The animal model used here reflects a lesion with a sharp transection of a previously fully intact tendon and lack of surgical repair. While this does not resemble a routine situation frequently seen in the clinic, it nevertheless provides a high level of reproducibility and omits confounding factors such as tissue pre- damage by preceding inflammation. Therefore, we consider this model a valuable tool for the investigation of early remodeling processes in tendons that will also provide valuable insights in tendon scar formation and healing. Further, the animal model used may not be suitable for investigating structural and biomechanical improvement of tendons for an extended period after surgery. We and others $[12,30]$ observe the formation of chondrified areas within the defect area after 28 days, which eventually result in the formation of irreversible ectopic mineralization in the neotissue, ultimately hampering biomechanical analysis.

Another aspect that needs to be considered is the fact that Bevacizumab is a humanized antibody and is therefore potentially immunogenic in non-human applications. However, we could show that Bevacizumab is detectable in the tendon tissue several days after injection (Fig. 2b-e). There is an ongoing debate on the effectiveness of Bevacizumab on rat VEGF-A. It had been shown that Bevacizumab binds to rat VEGF-A, though with lower affinity compared to human VEGF [31]. Moreover, Bevacizumab has previously been shown to exert effects in rat models, further underlining its applicability in rodent animal models [32, 33]. Considering the relatively high concentration of Bevacizumab used in our experiment, the fact that Bevacizumab is a humanized antibody most likely does not compromise our findings.

\section{Conclusion}

In summary, we demonstrate that antiangiogenic treatment during early tendon healing is beneficial for tendon quality following injury and should be considered as an alternative to the hitherto strategies promoting angiogenesis for resolving tendinopathies.

\section{Acknowledgements}

This work was supported by grant Nr.E-14/20/107-TEM from the Paracelsus Medical University Research Fund (HT and FS) and by the German Research Foundation (FOR2240; HE 6743/2-1 and HE 6743/3-1 to LMH). PMU-FFF R-15/02/067-KAS and Adele Rabensteiner Foundation for AK-E.

\section{Disclosure Statement}

No conflict of interests exists. 


\section{Cellular Physiology Cell Physiol Biochem 2018;46:1148-1158 \begin{tabular}{lll} 
DOI: 10.1159/000489057 & and Biochemistry & $\begin{array}{l}\text { O } 2018 \text { The Author(s). Published by S. Karger AG, Basel } \\
\text { www.karger.com/cpb }\end{array}$ \\
\cline { 2 - 3 }
\end{tabular}}

Tempfer et al.: Bevacizumab Improves Tendon Repair

\section{References}

1 Benjamin M, Ralphs JR: Tendons and ligaments--an overview. Histol Histopathol 1997;12:1135-1144.

- Elliott DH: Structure and Function of Mammalian Tendon. Biol Rev Camb Philos Soc 1965;40:392-421.

3 Josza L, Kannus P: Human Tendons - anatomy, physiology, and pathology. Human Kinetics, 1997.

4 Carr AJ, Norris SH: The blood supply of the calcaneal tendon. J Bone Joint Surg (Brit) 1989;71:100-101.

$>5$ Tempfer H, Traweger A: Tendon Vasculature in Health and Disease. Front Physiol 2015;6:330.

6 Lehner C, Gehwolf R, Ek JC, Korntner S, Bauer H, Bauer HC, Traweger A, Tempfer H: The blood-tendon barrier: identification and characterisation of a novel tissue barrier in tendon blood vessels. Eur Cells Mat 2016;31:296-311.

-7 Yang G, Rothrauff BB, Tuan RS: Tendon and ligament regeneration and repair: clinical relevance and developmental paradigm. Birth Defects Res C Embryo Today 2013;99:203-222.

-8 Boyer MI, Watson JT, Lou J, Manske PR, Gelberman RH, Cai SR: Quantitative variation in vascular endothelial growth factor mRNA expression during early flexor tendon healing: an investigation in a canine model. J Orthop Res 2001;19:869-872.

-9 Housner JA, Jacobson JA, Morag Y, Pujalte GG, Northway RM, Boon TA: Should ultrasound-guided needle fenestration be considered as a treatment option for recalcitrant patellar tendinopathy? A retrospective study of 47 cases. Clin J Sports Med2010;20:488-490.

10 Klein A, Loewenstein A: Therapeutic Monoclonal Antibodies and Fragments: Bevacizumab. Dev Ophtalmol 2016;55:232-245.

11 Wilgus TA, Ferreira AM, Oberyszyn TM, Bergdall VK, Dipietro LA: Regulation of scar formation by vascular endothelial growth factor. Lab Invest 2008;88:579-590.

-12 Korntner S, Kunkel N, Lehner C, Gehwolf R, Wagner A, Augat P, Stephan D, Heu V, Bauer HC, Traweger A, Tempfer H: A high-glucose diet affects Achilles tendon healing in rats. Sci Rep 2017;7:780.

13 Lui PP: Histopathological changes in tendinopathy--potential roles of BMPs? Rheumatology 2013;52:21162126.

14 Saltzman BM, Jain A, Campbell KA, Mascarenhas R, Romeo AA, Verma NN, Cole BJ: Does the Use of PlateletRich Plasma at the Time of Surgery Improve Clinical Outcomes in Arthroscopic Rotator Cuff Repair When Compared With Control Cohorts? A Systematic Review of Meta-analyses. Arthroscopy 2016;32:906-918.

15 Rosso F, Bonasia DE, Marmotti A, Cottino U, Rossi R: Mechanical Stimulation (Pulsed Electromagnetic Fields "PEMF" and Extracorporeal Shock Wave Therapy "ESWT") and Tendon Regeneration: A Possible Alternative. Front Aging Neuroscie 2015;7:211.

16 Mao WF, Wu YF, Yang QQ Zhou YL, Wang XT, Liu PY, Tang JB: Modulation of digital flexor tendon healing by vascular endothelial growth factor gene transfection in a chicken model. Gene Ther 2017;24:234-240.

17 Zhang F, Liu H, Stile F, Lei MP, Pang Y, Oswald TM, Beck J, Dorsett-Martin W, Lineaweaver WC: Effect of vascular endothelial growth factor on rat Achilles tendon healing. Plast Recontruct Surg 2003;112:16131619.

18 Kaux JF, Janssen L, Drion P, Nusgens B, Libertiaux V, Pascon F, Heyeres A, Hoffmann A, Lambert C, Le Goff C, Denoel V, Defraigne JO, Rickert M, Crielaard JM, Colige A: Vascular Endothelial Growth Factor-111 (VEGF111 ) and tendon healing: preliminary results in a rat model of tendon injury. Muscles Ligaments Tendons J 2014;4:24-28.

19 Hsieh CF, Alberton P, Loffredo-Verde E, Volkmer E, Pietschmann M, Muller PE, Schieker M, Docheva D: Periodontal ligament cells as alternative source for cell-based therapy of tendon injuries: in vivo study of full-size Achilles tendon defect in a rat model. Eur Cell Mater 2016;32:228-240.

-20 Dallaudiere B, Zurlinden O, Perozziello A, Deschamps L, Larbi A, Louedec L, Pesquer L, Benayoun Y, Silvestre A, Serfaty JM: Combined intra-tendinous injection of Platelet Rich Plasma and bevacizumab accelerates and improves healing compared to Platelet Rich Plasma in tendinosis: comprehensive assessment on a rat model. Muscles Ligaments Tendons J 2014;4:351-356.

21 DiPietro LA: Angiogenesis and scar formation in healing wounds. Current opinion rheumatol 2013;25:8791.

-22 Carmeliet P, Jain RK: Molecular mechanisms and clinical applications of angiogenesis. Nature 2011;473:298-307. 


\section{Cellular Physiology Cell Physiol Biochem 2018;46:1148-1158

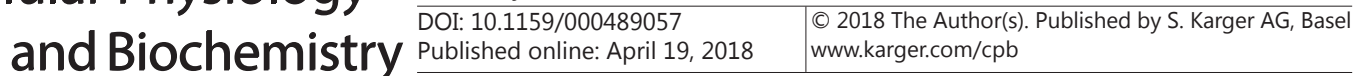 \\ Published online: April 19, $2018 \quad$ www.karger.com/cpb}

23 Presta LG, Chen H, O'Connor SJ, Chisholm V, Meng YG, Krummen L, Winkler M, Ferrara N: Humanization of an anti-vascular endothelial growth factor monoclonal antibody for the therapy of solid tumors and other disorders. Cancer Res 1997;57:4593-4599.

-24 Hos D, Koch KR, Bucher F, Bock F, Cursiefen C, Heindl LM: Serum eyedrops antagonize the anti(lymph) angiogenic effects of bevacizumab in vitro and in vivo. Invest Ophthalmol Vis Sci 2013;54:6133-6142.

-25 Koch KR, Refaian N, Hos D, Schlereth SL, Bosch JJ, Cursiefen C, Heindl LM: Autocrine impact of VEGF-A on uveal melanoma cells. Investigative ophthalmology \& visual science 2014;55:2697-2704.

26 Glade-Bender J, Kandel JJ, Yamashiro DJ: VEGF blocking therapy in the treatment of cancer. Expert Opinion Biol Ther 2003;3:263-276.

-27 Wu L, Martinez-Castellanos MA, Quiroz-Mercado H, Arevalo JF, Berrocal MH, Farah ME, Maia M, Roca JA, Rodriguez FJ, Pan American Collaborative Retina G: Twelve-month safety of intravitreal injections of bevacizumab (Avastin): results of the Pan-American Collaborative Retina Study Group (PACORES). Graefes Arch Clin Exp Ophthalmol 2008;246:81-87.

-28 Solomon SD, Lindsley KB, Krzystolik MG, Vedula SS, Hawkins BS: Intravitreal Bevacizumab Versus Ranibizumab for Treatment of Neovascular Age-Related Macular Degeneration: Findings from a Cochrane Systematic Review. Ophthalmology 2016;123:70-77 e71.

29 Berger MD, Lenz HJ: The safety of monoclonal antibodies for treatment of colorectal cancer. Expert Opin Drug Saf 2016;15:799-808.

-30 Gao Y, Zhang Y, Lu Y, Wang Y, Kou X, Lou Y, Kang Y: TOB1 Deficiency Enhances the Effect of Bone MarrowDerived Mesenchymal Stem Cells on Tendon-Bone Healing in a Rat Rotator Cuff Repair Model. Cell Physiol Biochem2016;38:319-329.

31 Irani Y, Scotney P, Nash A, Williams KA: Species Cross-Reactivity of Antibodies Used to Treat Ophthalmic Conditions. Invest Ophthalmol Vis Sci 2016;57:586-591.

-32 Di Cesare Mannelli L, Tenci B, Micheli L, Vona A, Corti F, Zanardelli M, Lapucci A, Clemente AM, Failli P, Ghelardini C: Adipose-derived stem cells decrease pain in a rat model of oxaliplatin-induced neuropathy: Role of VEGF-A modulation. Neuropharmacology 2017;131:166-175.

33 Sella R, Gal-Or O, Livny E, Dachbash M, Nisgav Y, Weinberger D, Livnat T, Bahar I: Efficacy of topical aflibercept versus topical bevacizumab for the prevention of corneal neovascularization in a rat model. Exp Eye Res 2016;146:224-232. 